\title{
MDCT evaluation of coronary artery calcification in Nepalese population undergoing CT thorax examination for non-cardiac pathology
}

\author{
Mukesh Kumar Gupta1, Raj Kumar Rauniyar², Naveen Kumar Pandey³, Deepak Kumar Yadav ${ }^{4}$ \\ ${ }^{1}$ Associate Professor, Department of Radiodiagnosis and Imaging, B.P. Koirala Institute of Health Sciences, Dharan, Nepal, ${ }^{2}$ Professor and \\ Head, Department of Radiodiagnosis and Imaging, B.P. Koirala Institute of Health Sciences, Dharan, Nepal, ${ }^{3}$ Assistant Professor, Department \\ of Internal Medicine, B.P. Koirala Institute of Health Sciences, Dharan, Nepal, ${ }^{4}$ Associate Professor, School of Public Health and Community \\ Medicine, B.P. Koirala Institute of Health Sciences, Dharan, Nepal
}

\section{A B S T R A C T}

Background: Coronary artery calcification (CAC) is frequently encountered as incidental findings during CT evaluation of thorax; however, little is known about its magnitude and association with atherosclerotic risk factors in Nepalese population. The purpose of this study was to evaluate the frequency of incidental CAC in patients undergoing standard thoracic CT examination for non-cardiac pathology and to correlate it with risk factors for coronary atherosclerosis. Methods: A hospital based prospective cross-sectional study was conducted on 216 patients over 8 month duration. The coronary arteries were evaluated for calcification on 16-slice MDCT and the frequency of CAC was correlated with atherosclerotic risk factors viz. age, sex, diabetes mellitus, hypertension, smoking and obesity. Results: Incidental CAC was seen in $72(33.3 \%)$ of total 216 patients. The rates of CAC below 40, 40-60 and above 60 years age group were $0 \%, 31.6 \%$ and $43.9 \%$ respectively. The frequency of CAC in male and female were $35 \%$ and $31.2 \%$ for all ages, $43.6 \%$ and $20 \%$ for $40-60$ years group, and $35.2 \%$ and $61.1 \%$ for above 60 years age group respectively. The frequency of CAC was higher in patients with diabetes mellitus (47.5\%), hypertension (42.3\%), smoking (43\%) and obesity (38.9\%). Conclusion: Incidental CAC was seen in $33.3 \%$ of the patients and it had significant association with advancing age, diabetes mellitus, hypertension and smoking. Rate of CAC was significantly higher in male for 40-60 years group but the frequency drastically increased in female for above 60 years group.

Key words: Coronary artery atherosclerosis, Coronary artery calcification, Incidental, Multidetector computed tomography

\section{INTRODUCTION}

Coronary artery disease, the most common form of heart disease, is the single most important cause of premature death globally. It is almost always due to atheroma and its complications, particularly thrombosis. The true frequency of atherosclerosis is difficult, if not impossible, to accurately determine because it remains predominantly silent and asymptomatic. Because arterial calcification almost always represents atherosclerosis, early detection of coronary artery calcification is of utmost importance in predicting and preventing myocardial infarction. A variety of imaging tools have been used to detect coronary artery calcification which includes plain chest radiography, fluoroscopy, echocardiography, CT scan and more recently endovascular ultrasonography. Plain chest radiography and fluoroscopy have the lowest sensitivity. CT scan is a highly sensitive tool for detection of coronary calcium and is being promoted as a noninvasive means for screening and diagnosis of coronary artery disease. ${ }^{1}$ Successive generations of CT technology i.e. conventional CT, electron beam CT (EBCT) and multidetector CT (MDCT) have been applied to cardiac imaging for detecting coronary calcification. Conventional CT scan has certain drawbacks e.g. longer scanning time 
with resultant motion artifacts, breathing misregistration, volume averaging and inability to quantify the plaque burden. ${ }^{2}$ Although EBCT is the standard technique and modality of choice for detection and scoring of CAC, it has limited availability and high cost. Due to recent advancement and tremendous development in the technology, MDCT has been shown to be equivalent to EBCT for detection and scoring of coronary calcification in current practice. ${ }^{3}$ The potentially important aspects of early detection of coronary artery atherosclerosis by MDCT in subclinical stage may be modification of cardiac risk factors, early treatment strategy and patient education on behavior modification with adoption of a healthier lifestyle so that catastrophic event can be prevented thus reducing the morbidity and mortality. Another important application of CT in quantification of coronary calcification may be monitoring of the response of the total atherosclerotic plaque burden to lipid-lowering drugs. ${ }^{3}$ The purpose of this study was to evaluate the frequency of incidental coronary artery calcification in patients undergoing standard thoracic CT examination for non-cardiac pathology and to correlate it with risk factors for coronary artery disease.

\section{METHODS}

A hospital based prospective cross-sectional study was carried out on 216 consecutive patients at B.P. Koirala Institute of Health Sciences (BPKIHS), Dharan, Nepal over a period of 8 months from March 2013 to October 2013. Informed consent was taken from all the patients. Approval of ethical clearance was obtained from the institutional ethical review board (IERB), BPKIHS, Dharan, Nepal. As it was a time bound study, sample size comprised of all the patients who underwent CT thorax during the study period and who fulfilled the inclusion criteria. All the patients referred for thoracic CT examination for evaluation of respiratory and non-cardiac pathology were included in the study. Patients with known cardiac disease including prior coronary artery bypass surgery (CABG), artificial valves, stents, or pacemakers were excluded from the study. Nondiagnostic scans with poor image resolution due to cardiac or respiratory movements were also excluded. The scans were performed by taking $7.5 \mathrm{~mm}$ contiguous sections from the apex to the base of the thorax, using a standard thoracic protocol, on a Hitachi 16-slice MDCT scanner without cardiac gating. The raw data were subsequently processed and multiplanar (MPR) and three dimensional (3-D) reconstructions were done from acquired thin axial sections. CT protocols were modified on the basis of the nature of the pathology for which the patient was referred. Intravenous contrast agent was administered wherever required depending on the nature of the pathology. Coronary artery calcification was only diagnosed when it was apparent on an artifact free scan and clearly visible on standardized soft tissue window in the anatomical sites of either left main, circumflex, anterior descending or right coronary artery. Calcification was defined as a visible hyper dense focus with Hounsfield unit (HU) of more than 130 over an area of $\geq 1$ sqmm. There was an awareness of the potential drawback that the calcification in the aortic valve, aortic root, mitral annulus and pericardium could mimic coronary artery calcification. The relevant clinical data i.e. age, sex, height, weight, smoking habit, hypertension and diabetes mellitus were recorded on a structured proforma. Obesity was defined as BMI value of $\geq 30 \mathrm{~kg} / \mathrm{m}^{2}$. The frequency of CAC was correlated with the risk factors for coronary artery atherosclerosis viz. advancing age, gender, hypertension, diabetes mellitus, smoking and obesity to look for the association. Statistical analysis was performed using SPSS software. Chi-square test was used to test the significance of variables. Statistical significance was defined as p-value $<0.05$ with 95\% confidence interval.

\section{RESULTS}

A total of 216 thoracic CT scans were evaluated in this study for incidental coronary artery calcifications. The most common indication for CT thorax was lung malignancy followed by pulmonary tuberculosis, pneumonia, mediastinal mass, solitary pulmonary nodule (SPN), bronchiectasis, chest trauma, lung abscess, pleural mass, empyema thoracic, pulmonary AVM, achalasia cardia and diaphragmatic hernia in decreasing order of frequency. Of total 216 patients, $120(55.6 \%)$ were male and 96 (44.4\%) were female with male to female ratio of $1.25: 1$. The age ranged from 14 to 96 years with mean age of 58.4 years.

Among total 216 patients studied, 72 (33.3\%) patients had incidental coronary artery calcification on MDCT of which $42(58.3 \%)$ were male and $30(41.7 \%)$ were female with male to female ratio of $1.4: 1$. The age of the patients with coronary artery calcification ranged from 41 to 90 years with mean age of 65.5 years. The majority of the patients $(n=24)$ with coronary artery calcifications were between 61-70 years of age. Coronary artery calcification was not found in any of the patient below 40 years of age. The frequency of coronary artery calcification in 40-60 years and $>60$ years age group were $17 / 39$ (43.6\%) \& 25/71 $(35.2 \%)$ in male and $8 / 40(20 \%)$ and $22 / 36(61.1 \%)$ in female respectively.

Four coronary vessels viz. left main coronary artery (LMCA), left circumflex (LCX), left anterior descending (LAD) and right coronary artery (RCA) were evaluated for incidental calcification (Figures 1 and 2) on MDCT. Of total 72 patients with coronary artery calcification, 31 had single vessel 


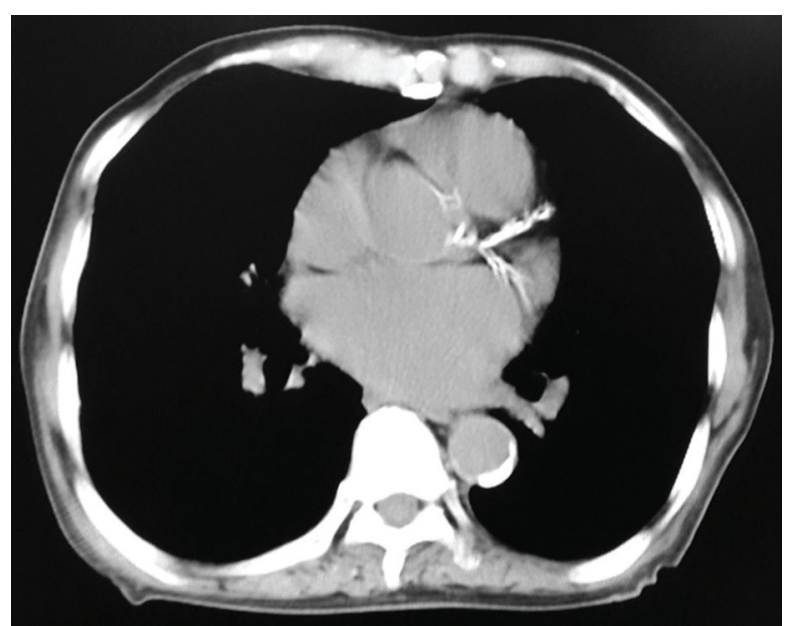

Figure 1: CT thorax at the level of heart shows calcifications in LMCA, LAD and LCX coronary arteries



Figure 2: CT thorax at the level of heart shows calcification in right coronary artery

calcification, 15 had two vessels calcifications, 17 had three vessels calcification and 9 had four vessels calcifications. The spectrum of coronary artery calcification in 72 patients detected on non-gated MDCT is shown in Table 1.

The relationship between coronary artery calcification and risk factors for ischemic heart disease is shown in Table 2. The MDCT showed rising trend of coronary artery calcification with advancing age of the patients. The frequency of calcification below 40, between 40-60 and above 60 years age group were $0 \%, 31.6 \%$ and $43.9 \%$ respectively and the difference was statistically significant. Although coronary artery calcification was more common in male patients for all ages, the difference was not statistically significant. Although the calcification was significantly higher in male for 40-60 years group, the rate increased drastically in female for $>60$ years group which was statistically significant. The frequency of coronary calcification in male and female were $35 \%$ and $31.2 \%$

\begin{tabular}{lcc}
$\begin{array}{l}\text { Table 1: Spectrum of coronary artery calcification } \\
\text { detected on MDCT in total } \mathbf{7 2} \text { patients }\end{array}$ \\
\hline Vessel(s) & $\begin{array}{c}\text { Frequency } \\
\text { (n=72) }\end{array}$ & Percentage \\
\hline Single artery calcification $(\mathrm{n}=31)$ & & \\
RCA & 10 & 13.88 \\
LCX & 9 & 12.5 \\
LAD & 6 & 8.33 \\
LMCA & 6 & 8.33 \\
Multiple artery calcification $(\mathrm{n}=41)$ & & \\
RCA, LAD, LCX & 11 & 15.27 \\
RCA, LMCA, LAD, LCX & 9 & 12.5 \\
LMCA, LAD, LCX & 6 & 8.33 \\
LAD, LCX & 4 & 5.55 \\
RCA, LAD & 4 & 5.55 \\
RCA, LMCA & 2 & 2.77 \\
RCA, LCX & 2 & 2.77 \\
LMCA, LAD & 2 & 2.77 \\
LMCA, LCX & 1 & 1.38 \\
\hline
\end{tabular}

$\begin{array}{lcccc}\text { Table 2: Association of coronary artery } & \\ \text { calcification with risk factors for ischemic heart } \\ \text { disease }\end{array}$

(P-value $>0.05$ ) for all ages, $43.6 \%$ and $20 \%$ ( $\mathrm{p}$-value $<0.05$ ) for $40-60$ years group, $35.2 \%$ and $61.1 \%$ (p-value $<0.05)$ for $>60$ years age group respectively. The frequency of coronary artery calcification was higher in patients with diabetes mellitus (47.5\%), hypertension (42.3\%) and smoking $(43 \%)$ which were statistically significant. Although the frequency of calcification was higher in patients with obesity $(38.9 \%)$, it was not statistically significant. 


\section{DISCUSSION}

Calcification of the arterial wall is intimately associated with mural injury and evolution of atherosclerotic plaque. Autopsy studies have shown the extent of coronary artery calcification to correlate with the plaque burden, severity of luminal stenosis and the frequency of ischemic heart disease. ${ }^{4,5} \mathrm{CT}$ scan is a highly sensitive technique for detection and quantification of coronary artery calcification. Absence of mural calcification at CT scanning has a high negative predictive value for ruling out coronary artery atherosclerosis and thus, its luminal stenosis. ${ }^{3}$ The negative predictive value of an EBCT calcium scan for significant stenosis (i.e. $\geq 50 \%$ diameter stenosis) of any major coronary vessel is about $90-95 \% .{ }^{2}$ Increasing amount of coronary calcium is directly associated with increasing likelihood of occlusive CAD and a high calcium score on EBCT may be consistent with moderate to high risk of a cardiovascular event within the next 2 to 5 years. ${ }^{6}$

Coronary artery calcification is often incidentally noted on chest $\mathrm{CT}$ in patients undergoing assessment for non-cardiac pathology. Various previous studies have reported the frequency of incidental coronary artery calcification on CT scan in the range of $22 \%$ to $46 \% .{ }^{7,8}$ In our study, MDCT showed incidental calcification in one or more coronary artery in $72(33.3 \%)$ of total 216 patients scanned for noncardiac pathology.

Presence of CAC on EBCT is nearly 100\% specific for coronary atherosclerosis but is not highly specific for obstructive disease, as both obstructive and non-obstructive atherosclerotic lesions can have intimal calcification. ${ }^{9}$ Literatures report that the sensitivity and specificity of EBCT in detecting coronary calcification in individuals with significant stenosis (>50\% narrowing) range from $85-100 \%$ and $45-47 \%{ }^{10-12}$ Masuda et al ${ }^{13}$ analyzed 161 patients who underwent cardiac CT as well as coronary angiography for evaluation of coronary artery disease and found that $90 \%$ of the patients with coronary calcification detected by CT had significant stenosis ( $>75 \%$ stenosis) on angiography, while $80 \%$ of the patients with significant stenosis on angiography had coronary calcification on CT scan. The sensitivity and specificity of CT coronary calcification for detection of significant stenosis were $65 \%$ and $87 \%$ respectively. ${ }^{13}$ Shemesh et $\mathrm{al}^{14}$ found that the helical CT demonstration of calcification had a sensitivity and specificity of $91 \%$ and $52 \%$ respectively when compared with the detection of significant coronary stenosis.

Advancing age, smoking, hypertension, hypercholesterolemia, diabetes mellitus and obesity are the major risk factors related to extent of coronary atherosclerosis and ischemic heart disease events. Pre-menopausal women have lower rates of CAD than men, although this sex difference disappears after the menopause. ${ }^{6,15}$ Our study showed significant association of $\mathrm{CAC}$ with advancing age, diabetes mellitus, hypertension and smoking which are the known major risk factors for coronary artery atherosclerosis. Although the rate of coronary calcification was higher in patients with obesity in our study, it was not statistically significant. We found that the rate of CAC was significantly higher in male for 40-60 years group but the frequency drastically increased in female for $>60$ years group.

\section{CONCLUSION}

Incidental CAC was seen in $33.3 \%$ of the 216 patients enrolled in our study and it had significant association with advancing age, diabetes mellitus, hypertension and smoking. Frequency of CAC was significantly higher in male for 40-60 years group but it drastically increased in female for above 60 years group. Although incidentally detected CAC may not have implication for the immediate clinical management of the patients, it may have impact on longterm clinical management and outcome. If CAC is found incidentally on CT scan, the patients should be assessed for ischemic features and risk factors for coronary artery disease so that preventive measures can be taken to avoid the catastrophic coronary events in future.

\section{ACKNOWLEDGEMENT}

The authors express appreciation to Dr. SR Niraula, Additional Professor, School of Public Health and Community Medicine, BPKIHS for assisting in statistical analysis.

\section{REFERENCES}

1. Wexler L, Brundage B, Crouse J, Detrano R, Fuster V, Maddahi J, et al. Coronary artery calcification: pathophysiology, epidemiology, imaging methods, and clinical implications. A statement for health professionals from the American Heart Association. Circulation 1996; 94(5):1175-1192.

2. Stanford $\mathrm{W}$ and Thompson BH. Imaging of coronary artery calcification. Its importance in assessing atherosclerotic disease. Radiol Clin North Am 1999; 37(2):257-272.

3. Schoepf UJ, Becker CR, Ohnesorge BM and Yucel EK. CT of coronary artery disease. Radiology 2004; 232(1):18-37.

4. Gowda RM and Boxt LM. Calcifications of the heart. Radiol Clin North Am 2004; 42(3):603-617.

5. Agatston AS, Janowitz WR, Hildner FJ, Zusmer NR, Viamonte MJ and Detrano R. Quantification of coronary artery calcium using Ultrafast computed tomography. J Am Coll Cardiol 1990;15(4):827-832.

6. O'Rourke RA, Brundage BH, Froelicher VF, Greenland P, Grundy SM, Hachamovitch $\mathrm{R}$, et al. American College of Cardiology/American Heart Association Expert Consensus document on electron-beam computed tomography for the 
diagnosis and prognosis of coronary artery disease. Circulation 2000; 102(1):126-140.

7. Callaway MP, Richards P, Goddard P and Rees $M$. The incidence of coronary artery calcification on standard thoracic CT scans. Br J Radiol 1997;70(834):572-574

8. Foley PW, Hamaad A, El-Gendi H and Leyva F. Incidental cardiac findings on computed tomography imaging of the thorax. BMC Research Notes 2010; 3:326. doi: 10.1186/1756-0500-3-326.

9. Budoff MJ, Achenbach S, Blumenthal RS, Carr JJ, Goldin JG, Greenland P, et al. Assessment of coronary artery disease by cardiac computed tomography: A scientific statement from the American Heart Association Committee on Cardiovascular Imaging and Intervention, Council on Cardiovascular Radiology and Intervention, and Committee on Cardiac Imaging, Council on Clinical Cardiology. Circulation 2006; 114(16):1761-1791.

10. Budoff MJ, Georgiou D, Brody A, Agatston AS, Kennedy J, Wolfkiel $\mathrm{C}$, et al. Ultrafast computed tomography as diagnostic modality in the detection of coronary artery disease: A multicenter study. Circulation 1996; 93(5):898-904.
11. Breen JF, Sheedy PFII, Schwartz RS, Stanson AW Kaufmann RB, Moll PP, et al. Coronary artery calcification detected with ultrafast CT as an indication of coronary artery disease. Radiology 1992; 185(2):435-439.

12. Fallavollita JA, Brody AS, Bunnel IL, Kumar K and Canty JMJ. Fast computed tomography detection of coronary calcification in the diagnosis of coronary artery disease: Comparison with angiography in patients $<50$ years old. Circulation 1994; 89(1):285-290.

13. Masuda $\mathrm{Y}$, Naito $\mathrm{S}$, Aoyagi $\mathrm{Y}$, Yamada Z, Uda T, Morooka N, et al. Coronary Artery Calcification Detected by CT: Clinical Significance and Angiographic Correlates. Angiology 1990; 41(12):1037-1047.

14. Shemesh J, Apter S, Rozenman J, LuskyA, Rath S, Itzchak Y, et al. Calcification of coronary arteries: Detection and quantification with double-helix CT. Radiology 1995;197(3):779-783.

15. Walker BR, Colledge NR, Ralston SH and Penman ID. Davidson's Principles and Practice of Medicine. 22 ${ }^{\text {nd }}$ Edition. Churchill Livingstone; 2014. pp 581.

Authors Contribution:

MKG - Concept and design of the study, data collection and analysis, statistical analysis, write up, literature search and review, manuscript preparation, critical review and editing; RKR - Concept and design of the study, literature review, interpretation of data, statistical analysis, manuscript preparation, review and editing; NKP - Concept and design, data collection and analysis, literature review, manuscript preparation, review and editing; DKY - Study design, data analysis and interpretation, statistical analysis, literature review, manuscript review and editing.

Source of Support: Nil, Conflict of Interest: None declared. 